\title{
Simulation and Effectiveness Analysis on One versus One Beyond Visual Range Air Combat
}

\author{
LIU Haoyu ${ }^{1}$, ZHANG Yunfei $^{1}$ and LI Shihao ${ }^{1}$ \\ ${ }^{1}$ School of Aeronautic Science and Engineering, Beihang University, Beijing 100191, China
}

\begin{abstract}
A kind of one versus one beyond visual range (BVR) air combat model has been established, which includes functional models of radar, missile and fighter and the process of several combat stages. Air combat effectiveness ratio (ACER) is defined to analyse the result. The $2^{\mathrm{k}}$ factor design method is used to design combat test case and analyses the influence of three factors (fighter stealth character, missile range and flight height) on ACER. Simulation result reveals that when RCS of one fighter is reduced from $0 \mathrm{dBm}^{2}$ to $-10 \mathrm{dBm}^{2}$ which cannot remarkably affect the opposition fighter's radar detection distance and missile launch distance, the RCS factor has small influence and the missile range factor has great influence. When RCS of one fighter is reduced from $-10 \mathrm{dBm}^{2}$ to $-20 \mathrm{dBm}^{2}$, the opposition fighter's radar detection distance will be reduced and lead the result of its missile launch distance be less than its missile range. Compared with the former case, the effect of RCS factor increases and the effect of missile range factor decreases. However, the effect of height is not significant.
\end{abstract}

\section{Introduction}

Modern air combat can be divided into within visual range (WVR) air combat and BVR air combat, and about $10 \mathrm{~km}$ is the dividing line of two cases. BVR air combat is a kind of air combat model that two hostile fighters are outside the visual range, detecting target through the airborne detection equipment and attacking each other with middle/long-range missiles. With the development of sensors and weapon systems, BVR air combat has become the main form of modern air combat [1-3].

Modern BVR air combat research is divided into two methods. Some of the literature [1-5] uses effectiveness assessment as the basic means, which is a static assessment method that establishes the advantage function of some important parameters (such as entry angle, speed, height, etc.), and the function is used to judge the results of air combat. The shortcoming of this method is relying on experience, and cannot accurately descript the random process of the modern air combat. Part of the literature [6-8] simulates the air combat through computer, which establishes the model of fighters based on kinematics and dynamics equation. Their shortcoming is that it is rough for the radar detection and track model and the missile attack process, affecting the assessment accuracy of results.

This paper establishes a relatively complete one versus one BVR air combat model, including radar search, track target, launch and guide missiles and damage judgment. Using $2 \mathrm{k}$ factor design method [9] to count the simulation data. Provide reference for the tactical decision, through analysis of stealth performance (radar cross section, RCS), flight height, missile range and other parameters on the impact of air combat.

\section{Radar detection and track model}

The purpose of radar simulation is to calculate the probability of detecting and tracking targets. During the BVR air combat period, the detection situation of airborne radar may be divided into 3 cases of upward, horizontal sight and overlooking. For the Pulsed Doppler (PD) radar, in the situation of upward and horizontal sight, detection performance will be affected only by noise. In the situation of overlooking, the ground clutter will reduce the detection performance of radar [10].

The detection performance of HPRF PD radar may be expressed in critical radar cross section [11]

$$
\sigma_{\mathrm{cr}}^{(c+n)}=\left\{\begin{array}{l}
\sigma_{\mathrm{cr}}^{(n)}=\frac{R^{4} L_{\alpha}}{C_{s 1} F^{4}}, \\
\text { upward and horizontal sight, } \frac{|\Delta V|}{V_{R}} \geq 1 ; \\
\sigma_{\mathrm{cr}}^{(n)}\left\{1+\frac{C_{S 2^{\gamma}}}{V_{R} H^{2}}\left[1-\left(\frac{\Delta V}{V_{R}}\right)^{2}\right]\right\}, \\
\text { overlooking, } \frac{|\Delta V|}{V_{R}}<1 ;
\end{array}\right.
$$


$R$ is the distance from the target, $C_{s 1 \text { and }} C_{s 2}$ is the $1 \mathrm{st}$ and 2nd radar system character constant, $V_{R}$ and ${ }^{H}$ is the speed and height of the aircraft. $(c+n)$ expresses the signal detection in condition of noise and clutter, and $(n)$ expresses only in noise.

In the case of other parameters keeping constant, the signal noise ratio (SNR) is proportional to the RCS of the target, and is inversely proportional to the critical RCS of the target. Therefore, the SNR for a target with RCS of $\sigma$ is

$$
\frac{S}{N}=\frac{\sigma}{\sigma_{\mathrm{cr}}^{(c+n)}}
$$

According to the relationship of detection probability with SNR, the detection probability to a target may be gotten for a given false alarm probability $\hat{p}_{f a}$.

When the radar has detected a signal, it can't be confirmed by one or twice scans if the signal is a target or not. The radar has to continuously scan the target to get several signals, avoiding these cases of detecting the signal of irrelevant target or environmental false target. The requirement is in $\mathrm{n}$ times of scan, the radar has to detect the target signal no fewer than $\mathrm{m}(\mathrm{m}<\mathrm{n})$ times. And the relation between $m$ and $n$ is

$$
m=1.5 \sqrt{n}
$$

Here $m$ and $n$ is respectively 3 and 5 . It means that signal has to be detected no fewer than 3 times within five times scan, then it can be said that the target is being tracked.

\section{One versus one air combat process}

One versus one air combat model was built on basis of following assumptions:

- Simplifying the fighter as a particle, and red fighter and blue fighter are flying in parallel flight path at a constant supersonic speed. After launching the missile, the fighter's speed decreases to subsonic velocity.

- When the distance between the target and the missile is less than terminal guidance distance, the missile enters the autonomous terminal guidance. Nevertheless, the terminal guidance is not simulated here; a constant kill probability is given for this stage.

- The air-to-air missile is also considered as a particle, which is with a constant speed.

\subsection{Simulation and data statistics}

In this simulation, the fighter tactical decisions of both sides are the same, which include searching, detection, tracking, launching missile and missile terminal guidance and target killed, etc.

If one side missile has killed the opposition fighter, but the latter has not launched its missile or guided its missile into the terminal guidance, it is judged that the former is alive and the latter is dead.
Air combat is a random process and it will be influenced by the probability of these combat process such as detection, tracking, hitting and killing, etc. For the same combat case, the simulation has to be executed for many times, and simulation results should be counted up to give the integrated results such as success probability of the red side, success probability of the blue side, success probability of the two sides and failure probability of the two sides.

The whole simulation flowchart of air combat is show in Figure 1, and the flowchart of fighter against module is shown in Figure 2

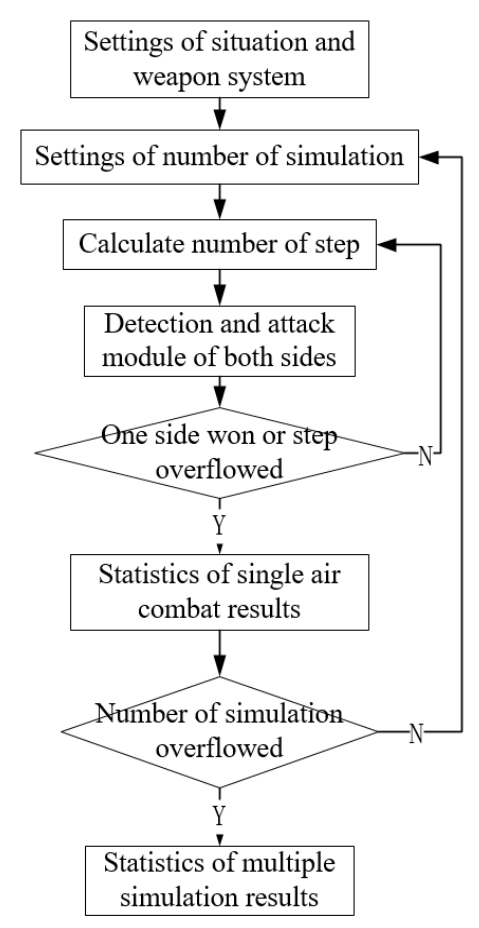

Figure 1. Whole simulation flowchart of air combat

\subsection{Radar search}

After the radar module calculates the detection probability $P_{d}$, the fighter should determine whether find the target or not using Monte Carlo method: firstly produce a random number $\alpha$ of $[0,1]$, and then if $\alpha<P_{d}$, it is considered that the fighter has found the target [12].

\subsection{Track target}

If the fighter has found the target, it need turn to the target and try to track it using pre-tracking method shown in Figure 3. $\mathrm{O}$ is the fighter; $\mathrm{T}$ is the target, and the flight direction is along vector $\boldsymbol{V}_{\boldsymbol{T}}$; the fighter does not flight to the target directly, but add a pre-tracking vector $k \boldsymbol{V}_{T} t_{(\mathrm{k}}$ is pre-tracking coefficient, $t$ is the radar sweep time), and then flights along vector OT'.

In the tracking state, if the detection result is not meet the discovery condition referred in part 2 , it means the fighter has lost the target and need return the radar search state. 


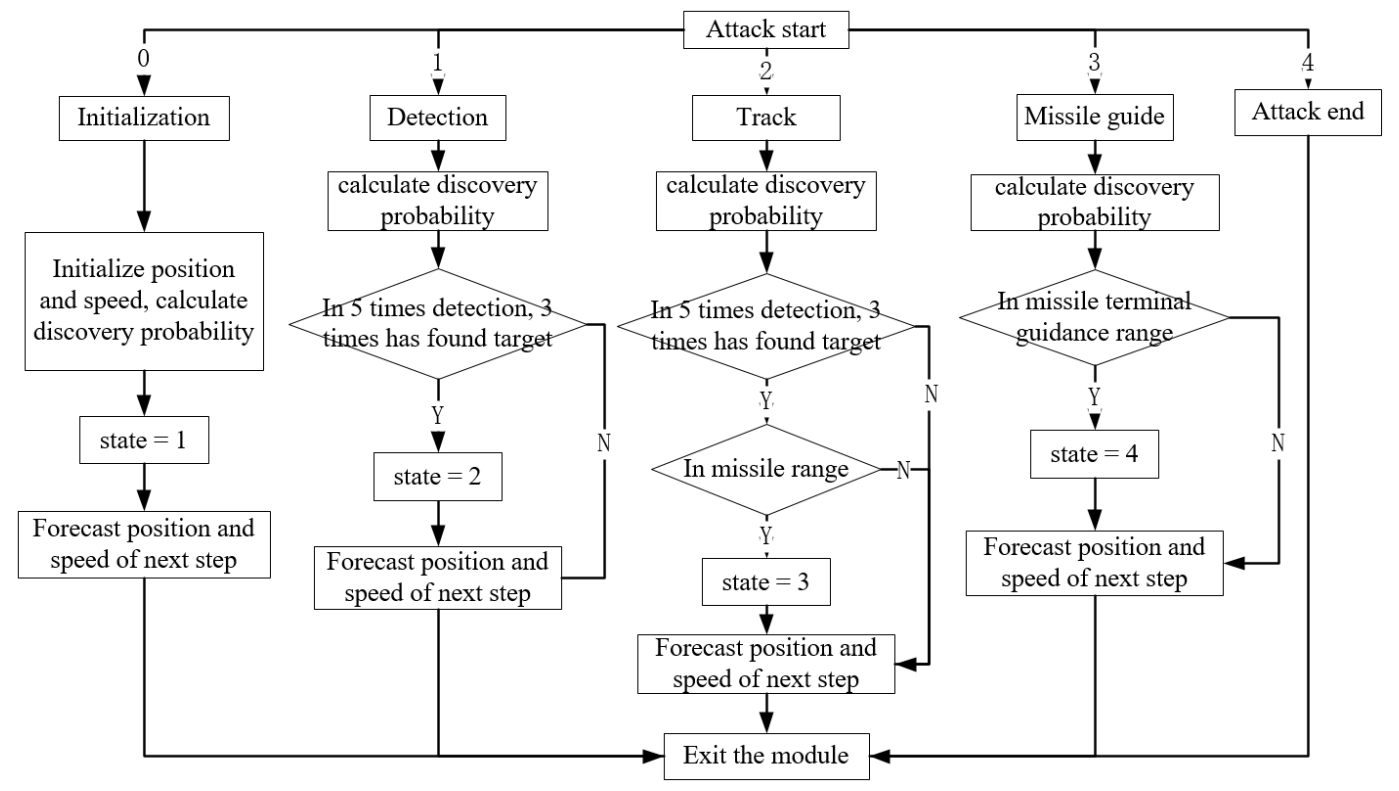

Figure 2. Flowchart of fighter against module

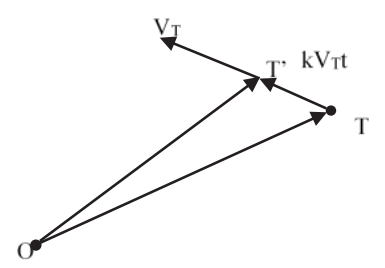

Figure 3. Pre-tracking method

\subsection{Launch and guide missiles}

When the fighter tracks the target, the fighter need launch missile if the distance between them is less than the range of missile. At this time, the airborne radar need continuously track the target and guide the missile flight to it. And the fighter should make the edge of scanning range turn to the target to avoid attack from enemy, which is shown in Figure 4. $\boldsymbol{V}_{\boldsymbol{O}}$ is the speed vector of the fighter

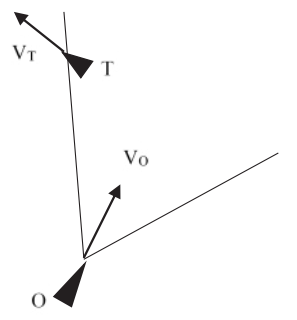

Figure 4. Missile guidance method

\subsection{Damage judgment}

After the missile enters terminal guidance, the fighter should maneuver to avoid the threat. Using Monte Carlo method to judge the result of air combat.

\section{Data statistics and analysis}

\section{$4.12^{k}$ factor design method}

$2 \mathrm{k}$ factor design method need select two values for each effect factor, and simulate all possible combinations of all effect factors and all values. If $\mathrm{k}=3$ ( 3 factors), there are 8 combinations (shown in Table 1). The sign factor $s_{i j}(+$, -) represent two values of factor $I_{i}$; response value $R_{j}$ is the value of simulation result.

Table 1. Combinations of factors

\begin{tabular}{|c|c|c|c|c|}
\hline $\begin{array}{c}\text { Combination } \\
j\end{array}$ & $\begin{array}{c}\text { Factor } \\
J_{1} \\
\operatorname{sign} s_{1 j}\end{array}$ & $\begin{array}{c}\text { Factor } \\
J_{2} \\
\operatorname{sign} s_{2 j}\end{array}$ & $\begin{array}{c}\text { Factor } \\
J_{3} \\
\operatorname{sign} s_{3 j}\end{array}$ & $\begin{array}{c}\text { Response } \\
\text { value } \\
\boldsymbol{R}_{j} \\
\end{array}$ \\
\hline 1 & - & - & - & $\mathrm{R}_{1}$ \\
\hline 2 & + & - & - & $\mathrm{R}_{2}$ \\
\hline 3 & - & + & - & $\mathrm{R}_{3}$ \\
\hline 4 & + & + & - & $\mathrm{R}_{4}$ \\
\hline 5 & - & - & + & $\mathrm{R}_{5}$ \\
\hline 6 & + & - & + & $\mathrm{R}_{6}$ \\
\hline 7 & - & + & + & $\mathrm{R}_{7}$ \\
\hline 8 & + & + & + & $\mathrm{R}_{8}$ \\
\hline
\end{tabular}

The main effect is the result $R_{j}$ 's average increment that the value of factor $I_{i}$ changes from - to + in the case of other factors unchanged, which is $J_{i}$ 's effect to result. And the calculation formula of main effect $e_{i}$ is

$$
e_{i}=\frac{1}{4} \sum_{j=1}^{8} s_{i j} R_{j}(i=1,2,3)
$$

The interactive effect of two factors is one factor's influence on the other factor's main effect, which is the relevance of the two factors. And the calculation formula of $e_{12}$ is.

$$
\begin{aligned}
e_{12} & =\frac{1}{2}\left[\frac{1}{2}\left(R_{8}-R_{6}+R_{4}-R_{2}\right)-\frac{1}{2}\left(R_{7}-R_{5}+R_{3}-R_{1}\right)\right] \\
& =\frac{1}{4}\left(R_{1}-R_{2}-R_{3}+R_{4}+R_{5}-R_{6}-R_{7}+R_{8}\right)
\end{aligned}
$$


The first part in the square bracket is the main effect of $I_{2}$ when $I_{1}$ 's value is + , and the second part is the main effect of $I_{2}$ when $J_{1}$ 's value is -. So $e_{12}$ is the increment of $I_{2}$ 's main effect when the value of factor $I_{i}$ changes from - to + , and it is easy to prove that $e_{12}=e_{21}$. Similarly,

$$
\begin{aligned}
& e_{13}=\frac{1}{4}\left(R_{1}-R_{2}+R_{3}-R_{4}-R_{5}+R_{6}-R_{7}+R_{8}\right) \\
& e_{23}=\frac{1}{4}\left(R_{1}+R_{2}-R_{3}-R_{4}-R_{5}-R_{6}+R_{7}+R_{8}\right)
\end{aligned}
$$

\subsection{Definition of air combat effectiveness ratio}

Define the air combat effectiveness ratio (ACER) of Red/Blue is

$$
R_{e x}(r / b)=\frac{P_{r}}{P_{b}}=\frac{P(\bar{r}, b)+P(\bar{r}, \bar{b})}{P(r, \bar{b})+P(\bar{r}, \bar{b})}
$$

$P(\bar{r}, b)$ is the probability that red fighter is alive and blue fighter is dead; $P(r, \bar{b})$ is the probability that red fighter is dead and blue fighter is alive; $P(\bar{r}, \bar{b})$ is the probability that both sides are alive.

The ACER of Red/Blue is the advantage of red fighter against the blue fighter. If the ratio is more than 1 , it means red fighter is in advantage; and if the ratio is less than 1 , blue fighter is in advantage.

\subsection{Results and analysis}

In order to avoiding the uncertainty brought by the change of RCS with the azimuth, we select sphere as the aircraft model. Air combat scene is that red fighter and blue fighter are flying head to head from the distance of $200 \mathrm{~km}$, and each simulation is repeated 10000 times. Some parameters are list in Table 2. Both sides use the same airborne radar, and

Table 3 shows the radar detection distance for targets with different RCS. RCS, flight height and missile range are chosen as the three factors, and the ACER of $\mathrm{Red} / \mathrm{Blue}$ is chosen as response value.

Table 2. Fighter and missile speed

\begin{tabular}{ll}
\hline fighter speed during searching $\left[\mathrm{km} \cdot \mathrm{s}^{-1}\right]$ & 0.3 \\
fighter speed after launching missile $\left[\mathrm{km} \cdot \mathrm{s}^{-1}\right]$ & 0.2 \\
missile speed $\left[\mathrm{km} \cdot \mathrm{s}^{-1}\right]$ & 1.0 \\
\hline
\end{tabular}

Table 3. Radar detection distance

\begin{tabular}{ccc}
\hline $\begin{array}{c}\text { RCS } \\
{\left[\mathbf{d B m}^{\mathbf{2}}\right]}\end{array}$ & $\begin{array}{c}\text { Upward and parallel sight } \\
{[\mathbf{k m}]}\end{array}$ & $\begin{array}{c}\text { Overlooking } \\
{[\mathbf{k m}]}\end{array}$ \\
\hline 0 & 134 & 96 \\
-10 & 75 & 54 \\
-20 & 42 & 30 \\
\hline
\end{tabular}

\subsubsection{Example 1}

For the air-combat scene settings shown in Table 4, using the method above calculate the ACER of Red/Blue and the effect (shown in Table 5).

According to the results, as to the main effect, missile range's is the largest, and height's is least. When the missile range increases from $50 \mathrm{~km}$ to $100 \mathrm{~km}$, the ACER increases 2.42 times; when RCS of Red/Blue increases from $0 /(-10) \mathrm{dBm} 2$ to $0 / 0 \mathrm{dBm} 2$, the ACER increases only 0.16 times; when the height of Red/Blue increases from $5 / 5(\mathrm{~km})$ to $10 / 5(\mathrm{~km})$, the ACER decreases 0.03

\begin{tabular}{|c|c|c|c|c|}
\hline \multirow{2}{*}{$\begin{array}{c}\text { Combat } \\
\text { case }\end{array}$} & \multicolumn{3}{|c|}{ Red/Blue } & \multirow[b]{2}{*}{ ACER } \\
\hline & $\begin{array}{c}R C S \\
{\left[\mathrm{dBm}^{2}\right]}\end{array}$ & $\begin{array}{c}\text { missile range } \\
R[\mathrm{~km}]\end{array}$ & $\begin{array}{l}\text { height } \\
\text { H[km] }\end{array}$ & \\
\hline 1 & $0 /-10$ & $50 / 50$ & $5 / 5$ & 0.80 \\
\hline 2 & $0 / 0$ & $50 / 50$ & $5 / 5$ & 1.01 \\
\hline 3 & $0 /-10$ & $100 / 50$ & $5 / 5$ & 3.28 \\
\hline 4 & $0 / 0$ & $100 / 50$ & $5 / 5$ & 3.39 \\
\hline 5 & $0 /-10$ & $50 / 50$ & $10 / 5$ & 0.80 \\
\hline 6 & $0 / 0$ & $50 / 50$ & $10 / 5$ & 0.98 \\
\hline 7 & $0 /-10$ & $100 / 50$ & $10 / 5$ & 3.24 \\
\hline 8 & $0 / 0$ & $100 / 50$ & $10 / 5$ & 3.36 \\
\hline
\end{tabular}
times.

Table 4. Settings and result of example 1

Table 5. Main effect and interactive effect

\begin{tabular}{ccc}
\hline \multirow{3}{*}{ Main effect } & $\boldsymbol{R} \boldsymbol{C S}$ & 0.16 \\
& $\boldsymbol{R}$ & 2.42 \\
& $\boldsymbol{H}$ & -0.03 \\
\hline \multirow{3}{*}{ Interactive effect } & $\boldsymbol{R} \boldsymbol{C S}-\boldsymbol{R}$ & -0.04 \\
& $\boldsymbol{R} \boldsymbol{C S}-\boldsymbol{H}$ & -0.01 \\
& $\boldsymbol{R}-\boldsymbol{H}$ & -0.01 \\
\hline
\end{tabular}

The reason that the main effect of RCS is small is: when the RCS of blue fighter decreases from $0 \mathrm{dBm} 2$ to $10 \mathrm{dBm} 2$ and the missile range is $50 \mathrm{~km}$ (combination 1 and 2), detection range for red radar is more than $50 \mathrm{~km}$, so it doesn't affect launch distance of red missile, but will affect the ACER for the difference of RCS; when the missile range is $100 \mathrm{~km}$ (combination 3 and 4), the variety of RCS will affect launch distance of red missile, but red fighter's launch distance is far greater than blue fighter's, so the ACER is not significantly affected by RCS.

\subsubsection{Example 2}

For the air combat scene settings shown in Table 6, using the method above calculate the ACER of Red/Blue and the effect (shown in Table 7).

According to the results, as to the main effect, RCS's is the largest, and height's is least, and the impact of RCS on ACER is significantly increased.

For cases that the red missile range is $50 \mathrm{~km}$, when blue fighter's RCS is $-10 \mathrm{dBm}^{2}$, the red radar's detection range of upward/overlook is $75 / 54 \mathrm{~km}$, which is larger than missile range, indicating that launch distance is not affected; when blue fighter's RCS is $-20 \mathrm{dBm}^{2}$, detection range of upward/overlook is $42 / 30 \mathrm{~km}$, and the launch increases by $10 \sim 20 \mathrm{~km}$. Moreover, the low RCS worsens 
target tracking conditions of red radar, so the exchange ratio of Red/Blue reduces by about 0.7 .

Table 6. Settings and result of example 2

\begin{tabular}{|c|c|c|c|c|}
\hline \multirow{2}{*}{$\begin{array}{c}\text { Combat } \\
\text { case }\end{array}$} & \multicolumn{3}{|c|}{ Red/Blue } & \multirow[b]{2}{*}{ ACER } \\
\hline & $\begin{array}{c}R C S \\
{\left[\mathrm{dBm}^{2}\right]}\end{array}$ & $\begin{array}{c}\text { missile range } \\
R[\mathrm{~km}]\end{array}$ & $\begin{array}{l}\text { height } \\
\text { H[km] }\end{array}$ & \\
\hline 1 & $-10 /-20$ & $50 / 50$ & $5 / 5$ & 0.31 \\
\hline 2 & $-10 /-10$ & $50 / 50$ & $5 / 5$ & 1.01 \\
\hline 3 & $-10 /-20$ & $100 / 50$ & $5 / 5$ & 0.31 \\
\hline 4 & $-10 /-10$ & $100 / 50$ & $5 / 5$ & 3.24 \\
\hline 5 & $-10 /-20$ & $50 / 50$ & $10 / 5$ & 0.31 \\
\hline 6 & $-10 /-10$ & $50 / 50$ & $10 / 5$ & 0.94 \\
\hline 7 & $-10 /-20$ & $100 / 50$ & $10 / 5$ & 0.31 \\
\hline 8 & $-10 /-10$ & $100 / 50$ & $10 / 5$ & 3.23 \\
\hline
\end{tabular}

Table 7. Main effect and interactive effect

\begin{tabular}{ccc}
\hline Main effect & $\boldsymbol{R} \boldsymbol{C S}$ & 1.80 \\
& $\boldsymbol{R}$ & 1.14 \\
& $\boldsymbol{H}$ & -0.13 \\
\hline \multirow{3}{*}{ Interactive effect } & $\boldsymbol{R} \boldsymbol{C S}-\boldsymbol{R}$ & 1.14 \\
& $\boldsymbol{R} \boldsymbol{C S}-\boldsymbol{H}$ & -0.01 \\
& $\boldsymbol{R}-\boldsymbol{H}$ & 0.02 \\
\hline
\end{tabular}

For the $100 \mathrm{~km}$-range missile of red side, the launch distance entirely depends on the radar detection range. So when the RCS of blue fighter reduced from $-10 \mathrm{dBm}^{2}$ to $20 \mathrm{dBm}^{2}$, detection range of upward/overlook reduced from $75 / 54 \mathrm{~km}$ to $42 / 30 \mathrm{~km}$, and the exchange ratio reduces by about 2.9 .

Therefore, the reason that the main effect of RCS is the largest is: the decrease of RCS reduces the radar detection distance, resulting in decrease of missile launch distance, as well as the low RCS worsens target tracking conditions, so the ACER reduced significantly.

The reason that the main effect of missile range is less than example 1 is: when blue fighter RCS is $-20 \mathrm{dBm}^{2}$, regardless of missile range, red fighter has to wait until detection range within $42 / 30 \mathrm{~km}$, so the two kinds of missile range has little impact on ACER; when blue fighter RCS is $-10 \mathrm{dBm}^{2}$, for the $100 \mathrm{~km}$-range missile, red fighter can attack when get the target $(75 / 54 \mathrm{~km})$, but for the $50 \mathrm{~km}$-range missile, red fighter has to wait even if it has found the target. So the combined effects of missile range are reduced. Therefore, the decrease of RCS reduced radar detection distance, so that missile range cannot fully play a role.

\section{Conclusion}

A kind of method of one versus one BVR air combat has been established. It is built for the method of radar searching, detection probability calculation and target tracking, and missile guidance and control. As a result, it is formed for the fighting simulation process on two opposition sides attacking alternately. The combat success probability and ACER are given by amount of combat simulations for several supposed combat scenarios.

According to simulation result, when RCS of one side fighter is reduced from $0 \mathrm{dBm}^{2}$ to $-10 \mathrm{dBm}^{2}$, therefore for the opposite side, the radar detection distance is affected slightly so that the missile launch distance is reduced unobviously. In such case the effect of RCS is small and the effect of missile range is great. When RCS of one side fighter is reduced from $-10 \mathrm{dBm}^{2}$ to $-20 \mathrm{dBm}^{2}$ that for the opposite side the radar detection distance is significantly reduced and the missile launch distance is shorter than missile range. Compared with the former case, the effect of RCS is increased and the effect of missile range is decreased. However, the effect of height is not significant.

It is revealed that the RCS reduction of one side fighter should make the opposite radar detection distance shorter than the missile range, so the opposite missile cannot play its full role, resulting that the effect of RCS on the combat success probability and ACER become significant.

\section{References}

1. ZHANG Hongbo, LI Guiying, Kong fane. Research on Situation Assessment in BVR Air Combat. EOC, 17, 4 (2010), 9-13

2. DONG Yanfei, WANG Liyuan, ZHANG Hengxi. Synthesized Index Model for Fighter Plane Air Combat Effectiveness. AAAS, 27, 6 (2006), 10851087

3. GAO Yong, XIANG Jinwu. New Threat Assessment Non-Parameter Model in Beyong-Visual-Range Air Combat. JSS, 18, 9 (2006), 2570-2572

4. ZHOU Siyu, WU Wenhai, ZHU Meng. Analysis of Air Combat Situation Assessment Based on Nonparametric Methods. ACT, 41, 4 (2011), 13-16

5. JamesS.McGrew, JonathanP.How. Air-Combat Strategy Using Approximate Dynamic Programming. AIAA-2008-6796

6. ZHENG Chang, DONG Wenhong, NIU Qinggong. Air Combat Construction and Emulation Design of Fighter Beyond Horizon. SEE, 29, 12 (2009), 135137

7. JU Chuanwen, YANG Xiuzhen. Study on Simulation of the Attacking Ability of Air to Air Missiles for Beyond Visual Range Targets. JSS, 16, 9 (2004), 2048-2051

8. KONG Xiangjun, SHANG Chongyang, GAO Zhenghong. Research on Simulation Model of Fighter Maneuver. JNPU, 25, 3 (2007), 331-335

9. DONG Xiaolong, SUN Jinbiao, SUN Naixiang. Simulation Experiment Analysis of Effectiveness Index of Air-combat Beyond-visual-range. JSS, 23, 11 (2011), 2321-2326

10. ZHANG Kao, ZHANG Yunfei, MA Dongli. Calculation and Analysis of Radar Stealth Performance of Aircraft (NDIP, Beijing, 1997)

11. ZHANG Yunfei. Radar Stealth Performance Calculation and Software Integration System for Aircraft (Beihang University, Beijing, 1998)

12. LI Jianping, WU Hong, WANG Weiping. Metamodel Method for Radar System to Detect and Track Target. JSS, 19, 3 (2007), 639-642 